Virginia Commonwealth University

VCU Scholars Compass

2009

\title{
Poly(styrene-co-vinylbenzylchloride-co- divinylbenzene) coated iron oxide: Synthesis and effects on size and morphology
}

\author{
M. D. Shultz \\ Virginia Commonwealth University \\ J.R. Marin \\ Gaiker Technology Centre \\ S. H. Naik \\ Virginia Commonwealth University \\ See next page for additional authors
}

Follow this and additional works at: http://scholarscompass.vcu.edu/chem_pubs

Cart of the Chemistry Commons

Schultz, M. D., Marin, J. R., \& Naik, S. H., et al. Poly(styrene-co-vinylbenzylchloride-co-divinylbenzene) coated iron oxide: Synthesis and effects on size and morphology. Journal of Applied Physics, 105, 07B318 (2009). Copyright (C) 2009 American Institute of Physics.

\section{Downloaded from}

http://scholarscompass.vcu.edu/chem_pubs/25

This Article is brought to you for free and open access by the Dept. of Chemistry at VCU Scholars Compass. It has been accepted for inclusion in Chemistry Publications by an authorized administrator of VCU Scholars Compass. For more information, please contact libcompass@vcu.edu. 
Authors

M. D. Shultz, J. R. Marin, S. H. Naik, J. Wilkins, J. M. Laza, J. L. Vilas, M. Rodriguez, N. Perez, and E. E. Carpenter 


\title{
Poly(styrene-co-vinylbenzylchloride-co-divinylbenzene) coated iron oxide: Synthesis and effects on size and morphology
}

\author{
M. D. Shultz, ${ }^{1}$ J. R. Marin, ${ }^{2}$ S. H. Naik, ${ }^{1}$ J. Wilkins, ${ }^{1}$ J. M. Laza, ${ }^{3}$ J. L. Vilas, ${ }^{3}$ \\ M. Rodriguez, ${ }^{3}$ N. Perez, ${ }^{3}$ and E. E. Carpenter ${ }^{1, a)}$ \\ ${ }^{1}$ Virginia Commonwealth University, Richmond, Virginia 23284, USA \\ ${ }^{2}$ Gaiker Technology Centre, Zamudio, Biscay, Spain \\ ${ }^{3} U P V / E H U, B^{\circ}$ Sarriena $s / n$, Leioa, Biscay, Spain
}

(Presented 12 November 2008; received 22 September 2008; accepted 7 January 2009; published online 24 March 2009)

\begin{abstract}
Iron oxide nanoparticles were coated with a polymer synthesized from the monomers of styrene, divinylbenzene, and vinylbenzylchloride. The resultant polymer microspheres from synthesis without nanoparticle loading are primarily monodispersed with a diameter of $3.45 \mu \mathrm{m}$ as measured by scanning electron microscopy. The addition of $1 \%$ nanoparticles by mass appears to decrease the size of the microspheres down to $2.04 \mu \mathrm{m}$ as well as increase the polydispersity. This trend is also seen to continue as you add more nanoparticles to the system going from $3.45 \mu \mathrm{m}$ with $0 \%$ nanoparticles down to below $1 \mu \mathrm{m}$ for $5 \%$ nanoparticles. This indicates that the particles are not just incorporated into the polymer matrix but act as nucleation sites to begin the polymerization process. The polymerization process was found to have no effect on the nanoparticles themselves as the magnetic characterization showed only a mass dilution in saturation when corrected by thermal gravimetric analysis. (C) 2009 American Institute of Physics. [DOI: 10.1063/1.3077222]
\end{abstract}

Functionalization of magnetic nanoparticles is a key step for their integration into mainstream applications. This has been accomplished by several different methods ranging from simple chelating ligands to large polymer shells. ${ }^{1-5}$ Polymer shells have a greater effect on particle size but can offer more stability when compared to some chelating agents. ${ }^{6}$ The use of polymers in nanoparticle functionalization also allows for the possibility of encapsulating or incorporating other components into a single composite for multiple functionality and various applications. An example of this would be in targeted drug delivery where nanoparticles and drugs can be encapsulated in the same polymer bead/matrix. ${ }^{7}$ In any case, further understanding of possible synthetic anomalies that arise from the inclusion of nanoparticles in polymer synthesis is of interest.

This work investigates the coating of iron oxide nanoparticles with poly(styrene-co-vinylbenzylchloride-codivinylbenzene) that is formed by precipitation polymerization. The divinylbenzene (DVB) acts as a cross-linking agent which gives greater mechanical properties to the magnetic nanocomposites, as compared to using styrene alone. Then the vinylbenzylchloride confers a hydrophilic group to the nanocomposites, which makes it possible for use in a wider range of applications. The vinylbenzylchloride was also used for two specific reasons. First, with the only difference to styrene being the chlorine at the paraposition relative to the vinyl group or polymerization site, there should be little change in the mechanical properties and a good distribution of vinylbenzylchloride throughout the polymer should be achieved. This could allow for the magnetic beads to be utilized in innovative column separation techniques that typi-

${ }^{a)}$ Electronic mail: ecarpenter2@vcu.edu. cally use polystyrene beads with an added magnetic component inside the column. The second reason for utilizing vinylbenzylchloride was to allow for the magnetic composite to be functionalized for multiple applications through nucleophilic substitution reactions at the chlorine site.

Iron oxide nanoparticles were synthesized by a polyol technique similar to those found in the literature. ${ }^{8,9}$ In this part of the synthesis, all chemicals were used as received. First, $0.4 \mathrm{~g}$ of iron(II) acetate and $1 \mathrm{~g}$ of sodium hydroxide were added to $25 \mathrm{~mL}$ of ethylene glycol. The reaction solution was quickly heated to reflux under rapid magnetic stirring for $1 \mathrm{~h}$. Then the reaction solution was allowed to cool to room temperature and subsequently poured into $50 \mathrm{~mL}$ of methanol. The iron oxide nanoparticles were then magnetically extracted and further washed several times with methanol. In order to prepare the particles for the polymerization coating process, two further washes and extractions were performed with anhydrous acetonitrile and sonication to remove surface water, methanol, or glycol. After the last acetonitrile wash, the particles were dried in a vacuum oven and kept under vacuum until used.

The polymer synthesis was carried out by similar methods used to produce poly(styrene-co-divinylbenzene) microspheres ${ }^{10}$ with the addition of vinylbenzylchloride in place of some styrene monomer. In this step of the synthesis, the vinylbenzylchloride and azobisisobutyronitril (AIBN) were used as received, but the styrene and DVB monomers have been purified by a $0.1 \mathrm{M}$ sodium hydroxide solution to remove the inhibitor. The polymer beads and iron oxide containing polymer beads (magnetic nanocomposites) were synthesized with monomer percentages of 30\% styrene, 25\% vinylbenzylchloride, and 45\% DVB. The iron oxide nanoparticles were added to $1 \mathrm{~mL}$ of monomer solution at mass percentages of $0 \%, 1 \%, 3 \%$, and $5 \%$, which was then placed 


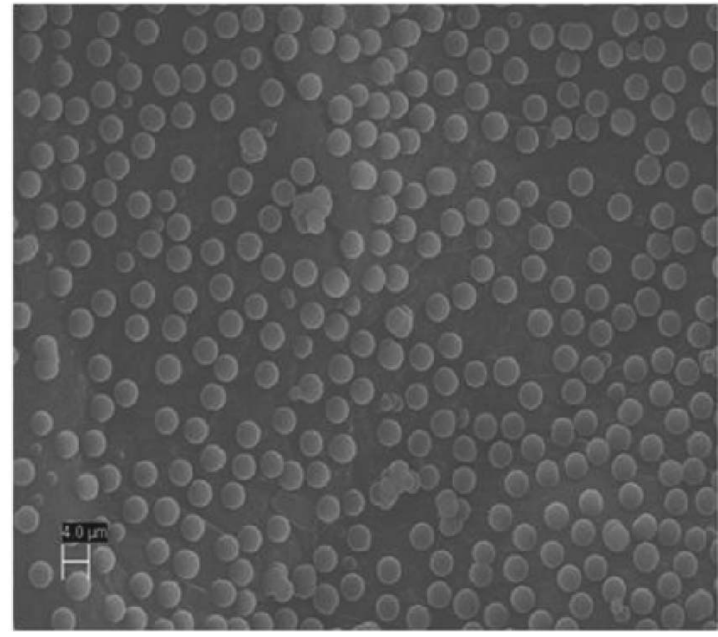

FIG. 1. SEM image of as synthesized polymer beads without nanoparticle loading.

under sonication to achieve a homogeneous dispersion of the particles in the monomer solution. Next, the monomer solution or monomer/particle solution was added to $50 \mathrm{~mL}$ of anhydrous acetonitrile, placed under $\mathrm{N}_{2}$ in a round bottom flask equipped with a reflux condenser, and stirred mechanically at a rate of $60 \mathrm{rpm}$. Then $20.0 \mathrm{mg}$ of AIBN was added to the reaction solution, followed by heating the solution to $75^{\circ} \mathrm{C}$ to initiate the polymerization. Once the temperature was stabilized at $75{ }^{\circ} \mathrm{C}$, the reflux condenser was capped with a balloon to maintain the reaction under $\mathrm{N}_{2}$, and the reaction was allowed to age for $24 \mathrm{~h}$. The reaction was then quenched in "cold" methanol $\left(\sim-20{ }^{\circ} \mathrm{C}\right)$ to precipitate the spherical polymer/nanocomposite beads. Vacuum filtration (magnetic separation for loaded samples) followed by further drying under vacuum was used to obtain the final product.

The resultant polymer microspheres from synthesis without nanoparticle loading are primarily monodispersed with a mean diameter of $3.45 \mu \mathrm{m}$, as measured by scanning electron microscopy (SEM) and seen in Fig. 1. The addition of $1 \%$ nanoparticles by mass appears to decrease the size of the microspheres down to a mean diameter of $2.04 \mu \mathrm{m}$ as well as increase the polydispersity, as seen in Fig. 2. The differ-

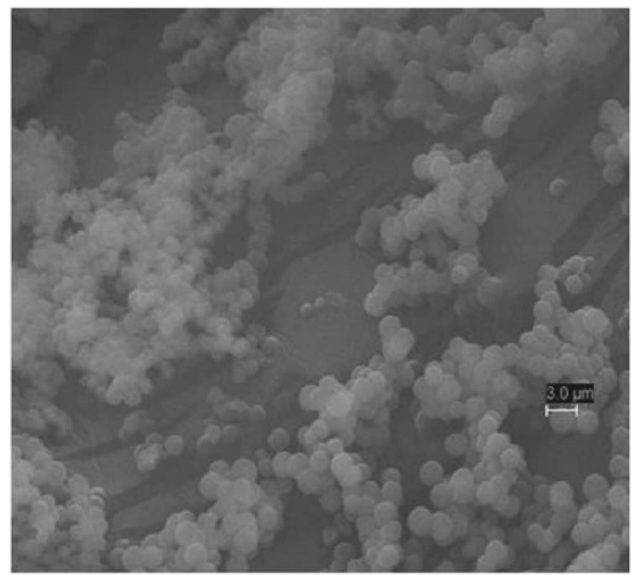

FIG. 2. SEM image of nanocomposite beads with $1 \%$ by mass nanoparticle loading in the polymer reaction.

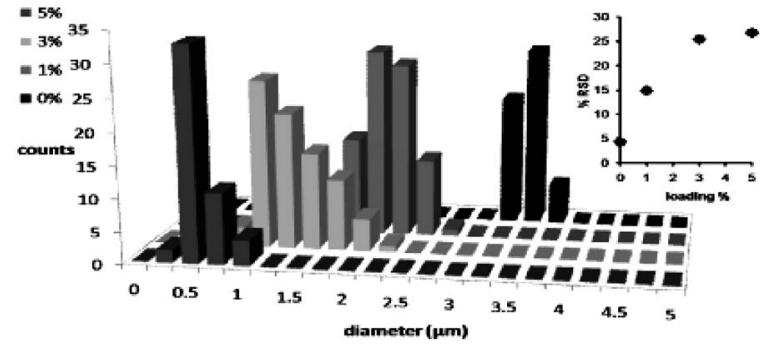

FIG. 3. Histograms of size measurements from SEM images of nanocomposites with varying nanoparticle loading percentages. (Inset) percent relative standard deviation plotted vs nanoparticle loading percentage.

ence in these two samples can be explained if the particles act as nucleation sites for the polymer chains. In the case without particles, the number of polymer chains should be somewhat proportional to the amount of initiator. Then with a uniform amount of chains being connected by cross-linker (DVB), uniformly sized spheres are achieved. When the nanoparticles are added, this gives a site for the monomers and forming polymer chains to orient or nucleate. This causes the DVB cross-linking agent to be used more quickly because the polymer chains are closer together, especially since the DVB has been found to be more reactive than the styrene monomer in the typical polymer synthesis. ${ }^{10}$ It also uses more monomers around the surface of the nanoparticles due to their high surface area, thus shortening the overall length of the polymer chains. This trend in size reduction is seen to continue as more nanoparticles are added to the system. The diameters of the spheres obtained are $3.45 \pm 0.15 \mu \mathrm{m}$ for $0 \%$ nanoparticles, $2.04 \pm 0.30 \mu \mathrm{m}$ for $1 \%, 1.32 \pm 0.34 \mu \mathrm{m}$ for $3 \%$, and $0.61 \pm 0.16 \mu \mathrm{m}$ for $5 \%$, and are shown in Fig. 3. The inset of Fig. 3 also shows that the percent relative standard deviation increases from 5\% to above $25 \%$, thus illustrating the increase in polydispersity as the nanoparticle loading increases.

Thermal gravimetric analysis (TGA) was then used to investigate the uniformity of the polymers formed. If increased nanoparticle loading would cause the DVB to be utilized more quickly, there should be evident differences in the decomposition profiles, which is indeed the case. As can be seen in Fig. 4, the $0 \%$ sample has a smooth decomposition

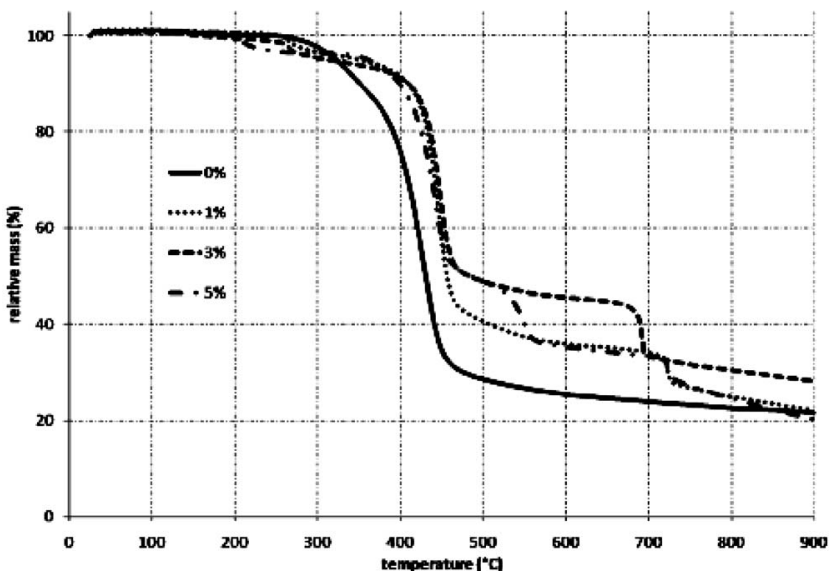

FIG. 4. TGA plot showing decomposition profiles for the nanocomposites and polymer beads. 


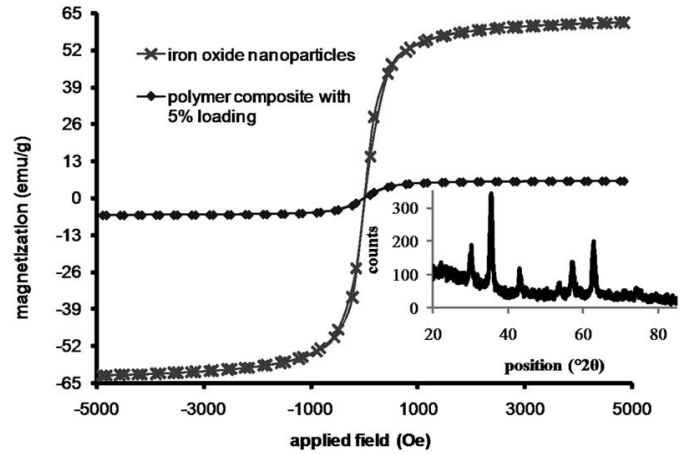

FIG. 5. Plot of magnetization vs applied field for the as synthesized iron oxide nanoparticles and the nanocomposite synthesized with 5\% loading of iron oxide nanoparticles. (Inset) powder x-ray diffraction pattern of as synthesized iron oxide nanoparticles.

and one primary decomposition temperature. As the loading is increased to $1 \%$, the first major decomposition temperature increases, which is indicative of higher levels of cross linking or greater density. There is also an addition decomposition event that is not present in the unloaded polymer bead. Increasing to $3 \%$ nanoparticles has a similar decomposition profile to $1 \%$, with slight differences in the later decomposition event. Then upon increasing to $5 \%$ loading, a third decomposition event occurs. This supports the idea that more nanoparticles would utilize the DVB more quickly, thereby causing a more nonuniform polymer to form.

Magnetic characterization by vibrating sample magnetometry was performed to validate that the composites synthesized were indeed magnetic and that the magnetization of iron oxide nanoparticles was retained postpolymerization. Figure 5 shows a hysteresis plot of the as synthesized iron oxide nanoparticles and the nanocomposite with 5\% nanoparticle loading, both measured at room temperature. As can be seen, the saturation magnetization of the iron oxide nanoparticles was around $62 \mathrm{emu} / \mathrm{g}$ and the saturation magnetization of the nanocomposite after polymerization was about 6 emu/g. Both samples showed a coercivity of roughly 10 Oe at room temperature. Utilizing the TGA data for mass correction of the nanocomposite sample yielded a magnetization of $60 \mathrm{emu} / \mathrm{g}$ of iron oxide in the sample. This value in magnetization, along with the confirmation of the spinel phase by powder x-ray diffraction seen in the inset of Fig. 5, gives evidence that the iron oxide synthesized is most likely maghemite and was unchanged during the polymerization process.

These results discussed indicate that the iron oxide nanoparticles are not just incorporated into the polymer matrix but act as nucleation sites to begin the polymerization process. Although the polymerization process was found to have no effect on the nanoparticles themselves, the nanoparticles had several effects on the resultant nanocomposite size and morphology by decreasing the diameter, increasing the polydispersity, and decreasing the uniformity of the polymer composition. These effects of nanoparticle loading reported give insight into parameters that may need to be adjusted in order to obtain similar polymer sizes, composition, and mechanical properties as nanoparticle loading is increased.

${ }^{1}$ N. A. D. Burke, H. D. H. Stover, and F. P. Dawson, Chem. Mater. 14, 4752 (2002).

${ }^{2}$ J. R. Jeong, S. J. Lee, J. D. Kim, and S. C. Shin, IEEE Trans. Magn. 40, 3015 (2004).

${ }^{3}$ P. Y. Keng, I. Shim, B. D. Korth, J. F. Douglas, and J. Pyun, ACS Nano 1, 279 (2007)

${ }^{4}$ J. Pyun, Polym. Rev. 47, 231 (2007).

${ }^{5}$ M. D. Shultz, S. Calvin, P. P. Fatouros, S. A. Morrison, and E. E. Carpenter, J. Magn. Magn. Mater. 311, 464 (2007).

${ }^{6}$ M. D. Shultz, J. U. Reveles, S. N. Khanna, and E. E. Carpenter, J. Am. Chem. Soc. 129, 2482 (2007).

S. Naik and E. E. Carpenter, J. Appl. Phys. 103, 3 (2008).

${ }^{8}$ S. Ammar, N. Jouini, F. Fievet, O. Stephan, C. Marhic, M. Richard, F. Villain, C. C. D. Moulin, S. Brice, and P. Sainctavit, J. Non-Cryst. Solids 345-346, 658 (2004).

${ }^{9}$ F. Fievet, J. P. Lagier, B. Blin, B. Beaudoin, and M. Figlarz, Solid State Ionics 32-33, 198 (1989).

${ }^{10}$ S. E. Shim, S. Y. Yang, and S. J. Choe, J. Polym. Sci., Part A: Polym. Chem. 42, 3967 (2004). 